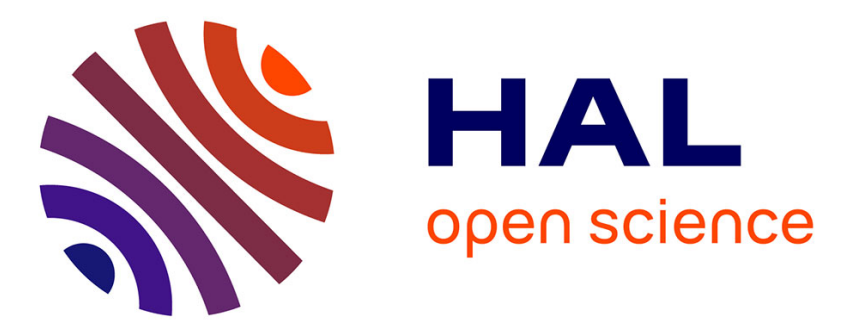

\title{
Disorder characterization of porous media and its effect on fluid displacement
}

Zhongzheng Wang, Kapil Chauhan, Jean-Michel Pereira, Yixiang Gan

\section{To cite this version:}

Zhongzheng Wang, Kapil Chauhan, Jean-Michel Pereira, Yixiang Gan. Disorder characterization of porous media and its effect on fluid displacement. Physical Review Fluids, 2019, 4 (3), 10.1103/PhysRevFluids.4.034305 . hal-02874913

\section{HAL Id: hal-02874913 \\ https://hal-enpc.archives-ouvertes.fr/hal-02874913}

Submitted on 19 Jun 2020

HAL is a multi-disciplinary open access archive for the deposit and dissemination of scientific research documents, whether they are published or not. The documents may come from teaching and research institutions in France or abroad, or from public or private research centers.
L'archive ouverte pluridisciplinaire HAL, est destinée au dépôt et à la diffusion de documents scientifiques de niveau recherche, publiés ou non, émanant des établissements d'enseignement et de recherche français ou étrangers, des laboratoires publics ou privés. 


\title{
Disorder characterization of porous media and its effect on fluid displacement
}

\author{
Zhongzheng Wang, ${ }^{1,2}$ Kapil Chauhan, ${ }^{1}$ Jean-Michel Pereira, ${ }^{2}$ and Yixiang Gan ${ }^{1, *}$ \\ ${ }^{1}$ School of Civil Engineering, The University of Sydney, NSW 2006, Australia. \\ ${ }^{2}$ Laboratoire Navier, UMR 8205, Ecole des Ponts ParisTech, IFSTTAR, CNRS, UPE, France.
}

\begin{abstract}
We investigate the effects of topological disorder and wettability on fluid displacement in porous media. A modified disorder index $I_{v}$ is proposed to characterize the disorder of porous media. By changing $I_{v}$, different displacement patterns (stable displacement and fingering) under the same flow condition and fluid property are obtained. We analytically demonstrate how increase in disorder promotes fingering due to uneven distribution of local capillary pressure. It is shown that the displacement efficiency for different wettability conditions and disorder well correlates with the distribution of local capillary pressure. A power law relation between fluid-fluid interfacial length and saturation of invading fluid is proposed by taking geometry into account, where the parameters in power law relation can be predicted by the capillary index, $I_{c}$, unifying the effects of topological disorder and wettability.
\end{abstract}

Keywords: Disorder, Fingering, Porous media, Interfaces, LBM, Displacement efficiency, Wettability.

\section{INTRODUCTION}

Displacement of multiphase fluids in porous media is involved in many industrial and natural processes, such as injection of $\mathrm{CO}_{2}$ into geological formations [1, 2], enhanced oil recovery $[3,4]$, remediation of contamination in aquifer systems [5], and water infiltration into soil [6]. Studies have been conducted with a focus on impacts of flow conditions and fluid properties on displacement of multiphase flows [7, 8], and effects of gravity [9] and wettability [10-12], while less attention has been paid on correlating the pore-scale disorder of porous media with the fluid displacement.

The displacement patterns, including capillary fingering $(\mathrm{CF})$, viscous fingering (VF), and stable displacement (SD), are primarily controlled by capillary number, $C a$, and viscosity ratio, $M$, between the defending and invading fluids [13-18]. When the invading fluid is more viscous than the defending fluid, i.e., $M<1$, the displacement patterns tend to shift from SD to $\mathrm{CF}$ with the decrease of $\mathrm{Ca}$, indicating the dominance of the interfacial tension. While in the case of $M>1$, increase of $C a$ modifies the flow toward VF. At the same time, wettability is also proven to play an important role: increasing contact angle of invading fluid results in more efficient displacement at all $C a$ [19-21], but when the contact angle exceeds a critical value, the trend is reversed due to corner flow [12].

Another important factor that influences fluids displacement is disorder of the porous media. For flows dominated by capillary effects (low $C a$ ), increase in disorder promotes fingering, leading to a transition from SD to $\mathrm{CF}[10,22]$. While for large $C a$ with $M>1$, high disorder modifies the viscous fingerings to become

\footnotetext{
* Corresponding author. Email: yixiang.gan@sydney.edu.au
}

more chaotic instead of having ordered patterns in regular media [13]. When both capillary and viscous effects are important, Holtzman [23] found that increase in disorder leads to higher interfacial area and lower displacement efficiency due to trapping. Holtzman [23] also offer subtle observations on effects of disorder by considering both capillary and viscous effects through scaling analysis [23]. However, many pore-scale simulation models assume quasi-static displacement [15], thus not capable of properly simulating dynamic mechanisms which are crucial even in slowly driven systems [21]. Despite a recent improvement of pore-scale simulation on ability of capturing non-local nature of interface dynamics [21], some processes during multiphase flow still remain un-captured such as droplet fragmentation [24]. In addition, due to the complex interplay among fluids properties, flow conditions, and topological features, the study of disorder effects on fluid displacement remain an active area of research, and attracts increased attention in the recent years with the help of development in microfluidics and advances in computational methods.

Multiphase flow in porous media has been studied both experimentally using micro-models $[12,15,16,25-27]$ and numerically by a range of simulation methods. PoreNetwork (PN) models, though computationally efficient, have limited predictive capability and accuracy due to simplification of pore geometries and/or flow equations [28-30]. Statistical models including Diffusion-Limited Aggregation (DLA), anti-DLA, and Invasion Percolation (IP) have been used to simulate VF, SD, and CF respectively. However, these "specialized" models cannot capture transitions between different regimes [18, 31]. Gridbased methods with interface tracking such as Volume of Fluid (VOF) method and Level Set (LS) method have been proposed to study multiphase flow in porous media [32-36]. However, they suffer from numerical instability at the interface when interfacial tension becomes dominant for micro-droplets [37]. In addition, they have only been applied to simple pore geometries due to relatively high computational costs. The Lattice Boltzmann method (LBM), as a meso-scale method, has been developed into a powerful tool for flow simulation in porous 
media [38-42]. Comparing to other numerical methods, the LBM is particularly suitable for pore-scale simulation of multiphase flows due to its ability of handling complex geometries and also being able to be massively parallelized. Therefore, it has been applied to study many problems in fluid mechanics[43-45].

In this paper, we investigate the effects of disorder and their coupling with wettability on fluid displacement in porous media. In order to better describe multiphase flow in porous media, a modified disorder index $I_{v}$ is proposed to characterize disordered geometry by reflecting the degree of fluctuation of local porosity. Samples are generated to have distinct values of $I_{v}$ for numerical simulations using Lattice Boltzmann method. Through controlling the disorder of geometry, we are able to produce different displacement patterns (stable displacement and fingering) under the same capillary number and viscosity ratio, providing new insights towards the conventional displacement phase diagram which is independent on system geometry. We demonstrate how increase in disorder collaborated with interfacial phenomena promote fingering due to uneven distribution of local capillary pressure. It is shown that for different wettability conditions and disorder, the displacement efficiency well correlates with the distribution of local capillary pressure. Finally, a power-law relation between fluid-fluid interfacial length and saturation of invading fluid is constructed. The parameters in power law relation can be predicted by the capillary index, $I_{c}$, which combines the effects of topological disorder and wettability.

\section{METHOD}

\section{A. Media generation and characterization}

Our geometry is a rectangular domain filled with circular obstacles to simulate the solid phase in porous media as as as shown in Fig. 1. The porosity of the medium, defined as the ratio of void area to total area in the 2D space, is controlled by varying the diameter of the obstacles. These obstacles are initially regularly placed on a triangular lattice.

To characterize the disorder of the medium, we use similar idea from Laubie et al. [46] but a different calculation method: instead of using fixed square meshing in their study of mechanical behavior of solid material (originally named as $I_{d}$ ), a Voronoi diagram is constructed for obtaining the local porosity, then a disorder index $I_{v}$ can be defined as the corrected standard deviation of local porosity:

$$
I_{v}=\sqrt{\frac{\sum_{n=1}^{N}\left(\phi_{n}-\bar{\phi}\right)^{2}}{N-1}},
$$

where $N$ is the number of obstacles in the domain, $\phi_{n}$ is the local porosity within the Voronoi cell, and $\bar{\phi}$ is the overall porosity of the medium.
Disorder is introduced by Monte-Carlo iterative movement of each obstacle with an apparent diameter $D_{\text {app }}=$ $\lambda D$ with $\lambda \in\left[1, D_{\max } / D\right]$, where $D$ is the original diameter of obstacle and $D_{\max }$ is the maximum diameter to achieve the maximum packing, e.g., ideally $\frac{\sqrt{3} \pi}{6}$ in $2 \mathrm{D}$, and its value depends on the system dimension and number of obstacles. Thus, $\lambda=1$ corresponds to a fully disordered system where no restriction is applied during perturbation except that overlap is avoided, while $\lambda=D_{\max } / D$ corresponds to a regular system where no obstacle is able to move since they are already in contact with each other according to their apparent diameter. Here, we focus on media with obstacles of same size. Periodicity is ensured in both horizontal and vertical direction for obstacle distribution and for the consequent Voronoi diagram in the periodic boundary (Fig. 1). During each time-step, perturbation is applied to each particle, after which the disorder index $I_{v}$ is calculated. This process stops when $I_{v}$ stabilizes around a certain value for given $\lambda$ (the fluctuations in $I_{v}$ are generally smaller than $5 \%$ at the end). Thus, $I_{v}=0$ corresponds to a perfectly ordered system, exhibiting no variation in local porosity; whereas large values of $I_{v}$ correspond to disordered systems by reflecting fluctuation of local porosity with respect to fully ordered one. We found that the disorder index $I_{v}$ has a monotonic correlation with $\lambda$ : as $\lambda$ decreases, $I_{v}$ increases and the system becomes more disordered. It is also found that the achievable maximum value of $I_{v}$ is dependent on the size of system domain, total number of obstacles, and the overall porosity. With our choice of a simulation domain with a length to width ratio $W / H=4$, and a periodic domain with $x \in[0.1299,3.9404]$ and $y \in[0,1]$ filled with 440 obstacles having a diameter of 0.06 (corresponding to an overall porosity of 0.6735$), I_{v} \in[0,0.08]$. Using this method, 5 geometries with distinct disorder have been generated for simulation with $I_{v}=[0,0.020,0.036,0.047,0.054]$ (see Fig. 2). Generally, with increasing $I_{v}$, the medium becomes more disordered and the variations in throat sizes becomes larger.

It is necessary to elaborate on reasons to introduce $I_{v}$ instead of directly using $\lambda$ as the disorder parameter $[10,23,25,47]$. Firstly, although a monotonic relation has been observed between $\lambda$ and $I_{v}$, from the definitions, $\lambda$ is a parameter for generating the geometry controlling the minimum distance between obstacles, while $I_{v}$ is the parameter for geometry characterization. Secondly, at the start of perturbation, the disorder of geometry can be quite different (usually in an increasing trend) for the same $\lambda$, until a sufficient number of iterations have passed. This is reflected by $I_{v}$ having an increasing tendency followed by fluctuation around a constant value, without which it is hard to define how many iterations are "sufficient". Finally, due to the the construction of Voronoi diagram for calculating $I_{v}$, each local porosity is dependent on its proximity of adjacent obstacles. Therefore, a large $I_{v}$ not only reflects the uneven spacial distribution of individual obstacles, but also implies the 
existence of dense clusters, which has strong influences on regional trapping as we will show in our results.

\section{B. Simulation method}

Standard Lattice Boltzmann method with D2Q9 lattice is used for our 2D numerical simulations, which includes streaming and collision steps as:

$$
f_{i}\left(\boldsymbol{x}+\boldsymbol{c}_{\boldsymbol{i}} \Delta t, t+\Delta t\right)=f_{i}(\boldsymbol{x}, t)+\Omega_{i}(\boldsymbol{x}, t),
$$

where $f_{i}$ is the density distribution function in $i$-th direction. In this study, Bhatnagar-Gross-Krook (BGK) approach was preferred to Multi Relaxation Time (MRT) one due to extra computational resources and complexity required for the latter [42]. The BGK collision operator is:

$$
\Omega_{i}(\boldsymbol{x}, t)=-\frac{f_{i}-f_{i}^{e q}}{\tau} \Delta t,
$$

which relaxes the distribution function towards an equilibrium $f_{i}^{e q}$ at a rate determined by the relaxation time $\tau$. The equilibrium distribution function is given by

$$
f_{i}^{e q}(\boldsymbol{x}, t)=\omega_{i} \rho\left[1+\frac{\boldsymbol{u} \cdot \boldsymbol{c}_{\boldsymbol{i}}}{c_{s}^{2}}+\frac{\left(\boldsymbol{u} \cdot \boldsymbol{c}_{\boldsymbol{i}}\right)^{2}}{2 c_{s}^{4}}-\frac{\boldsymbol{u} \cdot \boldsymbol{u}}{2 c_{s}^{2}}\right],
$$

where $\omega_{i}$ is the weight for the $i$ th direction. We use Shan-Chen multi-component model originally proposed by Shan and Chen [38], Shan and Doolen [39]. They introduced an inter-particle force as:

$$
F^{S C(\sigma)}(\boldsymbol{x})=-\psi^{(\sigma)}(\boldsymbol{x}) \sum_{\tilde{\sigma} \neq \sigma} G_{\tilde{\sigma} \sigma} \sum_{i} \omega_{i} \psi^{(\tilde{\sigma})}\left(\boldsymbol{x}+\boldsymbol{c}_{\boldsymbol{i}} \Delta t\right) \boldsymbol{c}_{\boldsymbol{i}} \Delta t,
$$

where $\psi^{(\sigma)}$ is the "effective" density function for $\sigma$ component, and $G$ is a simple scalar that controls the strength of the interaction. To model immiscible fluids, the interaction strength $G$ must be positive, simulating the repulsive force between different components. By adopting different fictitious wall densities, contact angles can be tuned [48]. Regularized boundary condition proposed by Latt and Chopard [49] is adopted to achieve second order accuracy. For more detailed information about Lattice Boltzmann method, we refer to Mohamad [50] and Kruger et al. [42]. We choose a mesh size of 800 x $3200 \mathrm{lu}^{2}$ (lu: lattice unit) for the simulation area such that at least 10 lattices are in between the smallest throat to ensure the grid is fine enough [51]. Each time after the generation of media, the minimum throat distance $r_{\min }$ is determined. To ensure minimum number of lattices $N_{\min }$ along $r_{\text {min }}$, the number of lattices required in vertical direction for the simulation domain is calculated as $M_{v}=\frac{N_{\min }}{r_{\min }} \times H$, and $M_{h}=4 \times M_{v}$ for horizontal direction, where $H$ is the height of the simulation domain shown in Fig.1. Note that this method assumes $r_{\min }$ and principal directions can be aligned. For example, with $N_{\min }=10$ and $r_{\min }=0.02$, the mesh needs to be (at least) $500 \times 2000$. Finer mesh is required as the topological disorder increases(reduce in apparent diameter leads to smaller possible $r_{\text {min }}$ ). After examining all possible cases, we finally choose a mesh of $800 \times 3200$ to ensure $N_{\min }=10$ for all simulation cases. The kinematic viscosities for both fluids are $0.1667 \mathrm{lu}$. The invading and defending fluids have densities of 1 and $0.8 \mathrm{lu}$, respectively, leading to a viscosity ratio $M=0.8$. The interfacial tension can be calculated using Young-Laplace equation, which is $0.2152 \mathrm{lu}$. The invading fluid is injected from the left with a constant velocity of $V_{\mathrm{inj}}=0.005 \mathrm{lu}$, leading to a capillary number $C a=\frac{V_{\mathrm{inj}} \mu_{\text {def }}}{\gamma}=0.0031$. The pore scale Reynolds number is less than 10 . The outlet pressure at the right end is set to be a constant of $0.2667 \mathrm{lu}$. Periodic boundary condition is applied at top and bottom of the simulation area. Overall, 25 simulations are carried out for 5 different disorder $\left(I_{v}=0\right.$, $0.020,0.036,0.047,0.054)$ and 5 different contact angle $\left(\theta=35^{\circ}, 62^{\circ}, 89^{\circ}, 109^{\circ}, 128^{\circ}\right)$.

\section{RESULTS AND DISCUSSION}

The displacement patterns for different wettability conditions and topological disorder are shown in Fig. 3. These are qualitative demonstration of effects of wettability and disorder on fluid displacement in porous media. Note that these snapshots correspond to the final stage of simulation, which is when the invading fluid reaches the right end of the periodic boundary (see Fig. 1). Generally, stronger fingering and larger trapped area of defending fluid are observed when the medium becomes more disordered (increasing $I_{v}$ ) and more hydrophobic (increasing $\theta$ ), implying a less efficient displacement, consistent with previous observations [9-12, 19-21, 23, 52]. Fig. 3 also demonstrates the "competition" between the destabilizing effect due to uneven distribution of capillary resistance and stabilizing effect from cooperative pore filling events, which have a higher occurrence when contact angle is small. To provide quantitative information about these patterns, the normalized fluid-fluid interfacial length, $L^{*}=\frac{\text { interfacial length }}{\text { width of geometry }}$, is calculated and plotted as a function of saturation of invading fluid $S$ for each time-step (Fig. 4). Note that in order to exclude the boundary effect at outlet we conduct representative volume analysis and found an area of interest with $L_{A O I}=3$ well captures the displacement data, so it is within this area (Fig. 1) our results are based on. Fig. 4 shows that the rates at which interfacial length increase are larger as the media become more disordered (following the direction of black arrow) for all wettability conditions. This dependence becomes stronger as the contact angle increases, which again can be explained by the stabilizing effect of wettability [21, 53]: trapping is mitigated by cooperative pore filling, or overlap, during displacement for disordered media (corresponding to the 4 collapsed curves for $\theta=35^{\circ}$ ). As the contact angle increases, this "mitigating effect" is reduced such that 


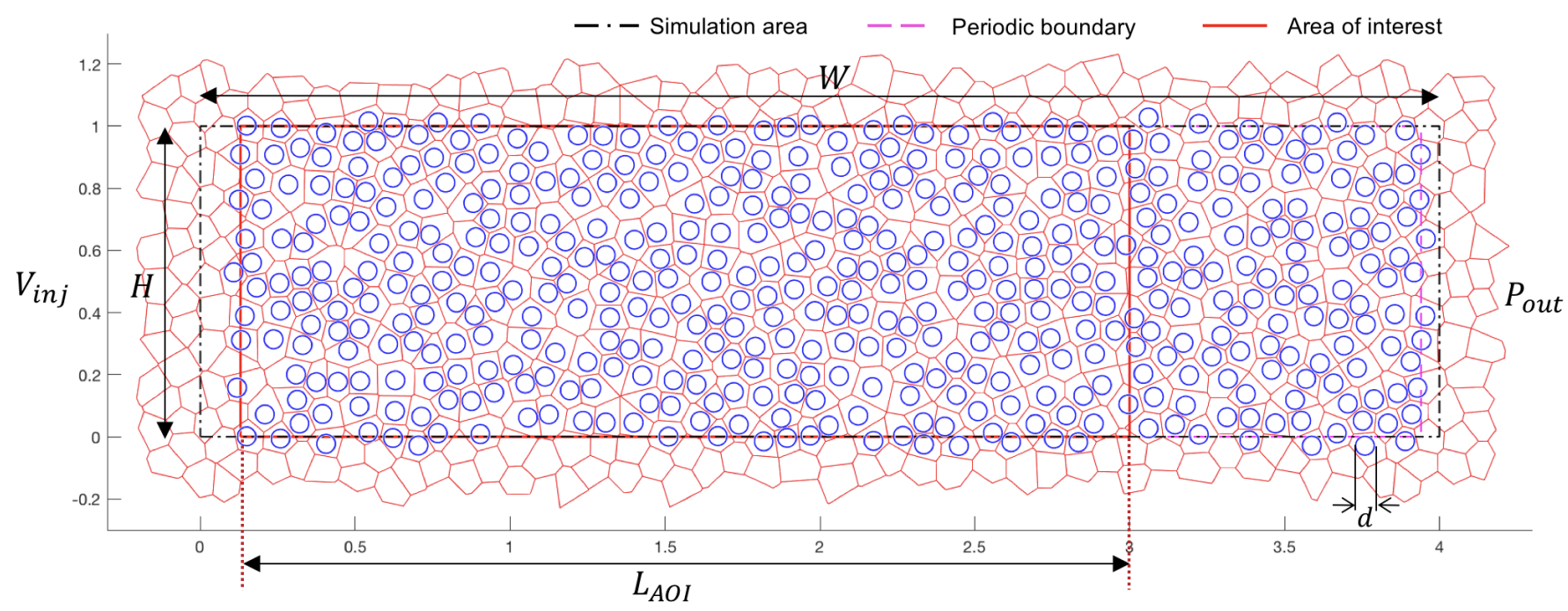

FIG. 1. Our porous medium is composed of 440 circular obstacles with diameter $d=0.06$, which are initially regularly placed on a triangular lattice. The area enclosed by black dot-dash line is the simulated area with injection width $H$ and longitudinal length $W$, while our results and analysis are based on the area of interest $\left(L_{A O I}\right)$, which is enclosed by red solid line. Geometrical periodicity in horizontal and vertical direction is ensured inside periodic boundary (purple-dashed line). The invading fluid is injected from the left with a constant velocity $V_{\text {inj }}$. The pressure at the right end is set to be constant $P_{\text {out }}$. Periodic boundary condition is imposed at top and bottom of the simulation area.
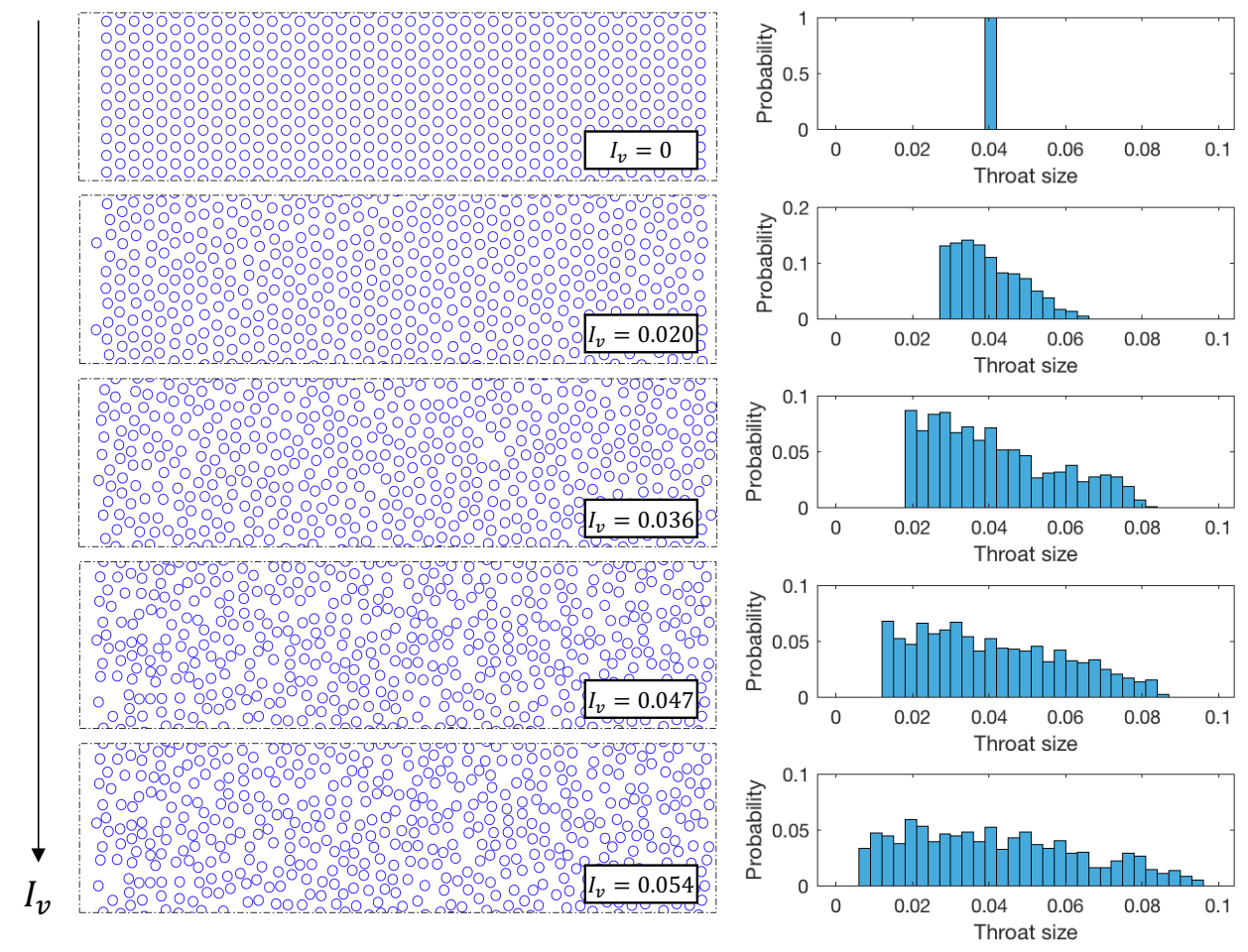

FIG. 2. Generated porous media and corresponding throat size probability distribution. From top to bottom, $I_{v}=0,0.020$, $0.036,0.047,0.054$.

more trapping events occur, leading to higher interfacial length. Furthermore, we also quantify (a) the residual saturation (Fig. 5A and Fig. 5C) and (b) the ratio of final interfacial length to saturation (Fig. 5B and Fig. 5D) as functions of $I_{v}$ and $\theta$ : increase in $I_{v}$ and $\theta$ leads to decrease in displacement efficiency and increase in interfacial length per unit of saturation. These results again demonstrate the combined impacts of topological disorder and wettability.

To analytically investigate how wettability and disor- 


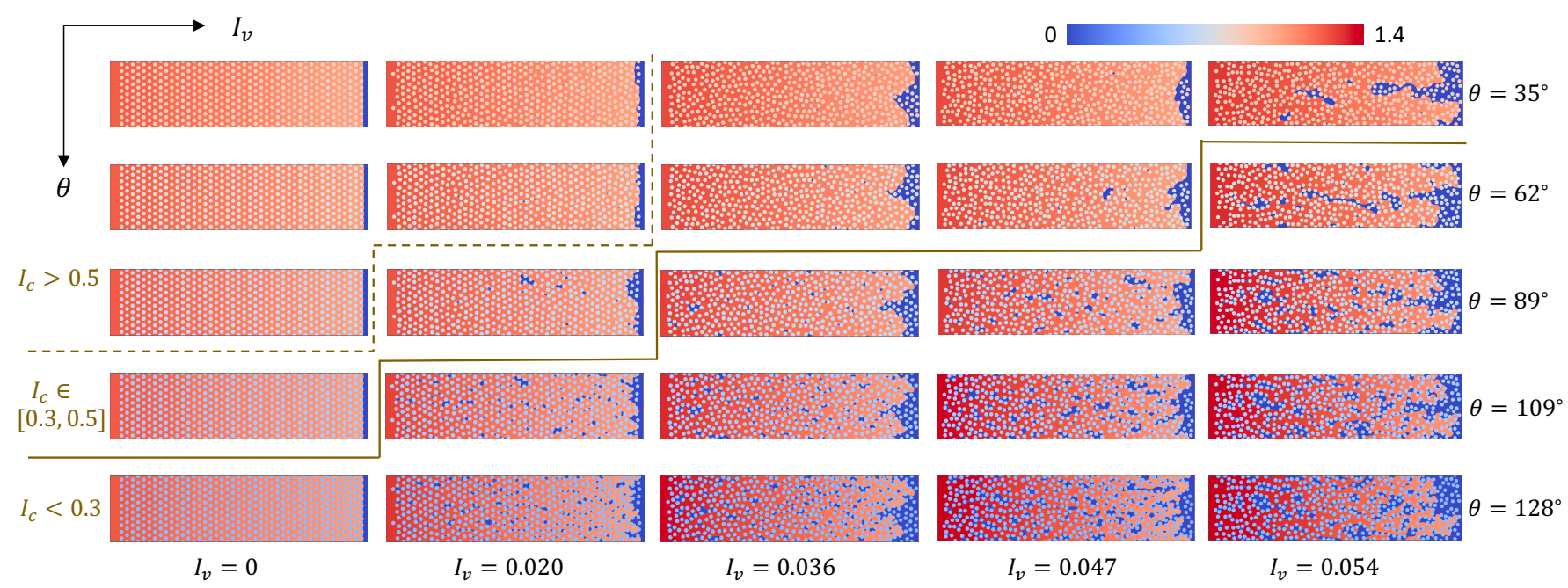

FIG. 3. Displacement patterns (from data of invading fluid) for different disorder (left to right, $I_{v}=0,0.020,0.036,0.047$, 0.054 ) and wettability (top to bottom, $\theta=35^{\circ}, 62^{\circ}, 89^{\circ}, 109^{\circ}, 128^{\circ}$ ). The color map represents the density of invading fluid in lattice unit. Note that in LBM, the density fields of invading and defending fluids are stored in separate matrices. Here only the matrix storing data of invading fluid is shown. The blue color (density of invading fluid being zero) stands for the location of defending fluid, and the density fluctuation represents the pressure variation.
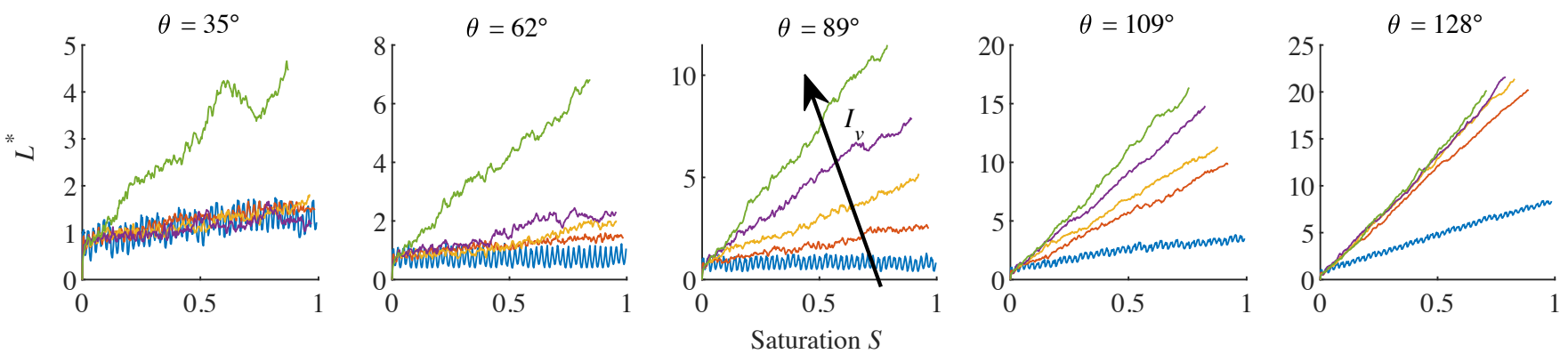

FIG. 4. Normalized fluid-fluid interfacial length $\left(L^{*}\right)$ as a function of saturation of invading fluid $(S)$ for different wettability and topological disorder. The black arrow indicates the direction of increasing disorder: $I_{v}=0,0.020,0.036,0.047,0.054$ corresponding to blue, orange, yellow, purple, and green curves respectively.

der cooperate together to influence fluid displacement, we start with the equation that contain the physics of fluid displacement in multiple throats as suggested by Lenormand et al. [15]:

$q=K_{1}\left(P-P_{c_{1}}\right)^{+}+K_{2}\left(P-P_{c_{2}}\right)^{+}+\ldots=\sum_{n=1}^{N} K_{n}\left(P-P_{c_{n}}\right)^{+}$,

where $q$ is is the total flow rate, $N$ is total number of throats, $K_{n}$ is the hydraulic conductance at local pore throat $n, P$ is the pressure difference of invading and defending fluids, and $P_{c_{n}}$ is the local capillary pressure providing resistance to the invasion of throat $n$. Locally, as long as $P$ is smaller than $P_{c_{n}}$, there is no displacement. Then, the pressure difference between invading and defending fluids increases as more invading fluid is injected, resulting in $P>P_{c_{n}}$ at throat $n$, leading to local throat invasion. Thus, in absence of viscous fingering, it is the uneven distribution of local capillary pressure that leads to uneven invasion of pores and consequent trapping and fingering, which ultimately affects the fluid-fluid interfacial length and displacement efficiency. To calculate the maximum allowable capillary pressure $P_{c, \max }$ before a throat is invaded, we consider 3 basic pore-scale mechanisms: "burst", "touch", and "overlap" [54]. Firstly, the equation for capillary pressure $P_{c}$ at throat $n$ in porous medium filled with circular obstacles of same diameter can be calculated by:

$$
P_{c_{n}}=\frac{2 \gamma \sin \left(\alpha+\theta-90^{\circ}\right)}{h_{n}-d \cos (\alpha)}
$$

where $\gamma$ is the interfacial tension, $h$ is the center-to-center distance of obstacles, $d$ is the diameter, and $\alpha$ is the filling angle shown in Fig. 6A. Then, we define that the throat is invaded if (i) the front of invading fluid comes in contact with the next obstacle (touch), or (ii) $\alpha$ reaches $\alpha_{\text {overlap }}$ corresponding to cooperative pore filling event (overlap), assuming that obstacles are placed on triangular lattices 

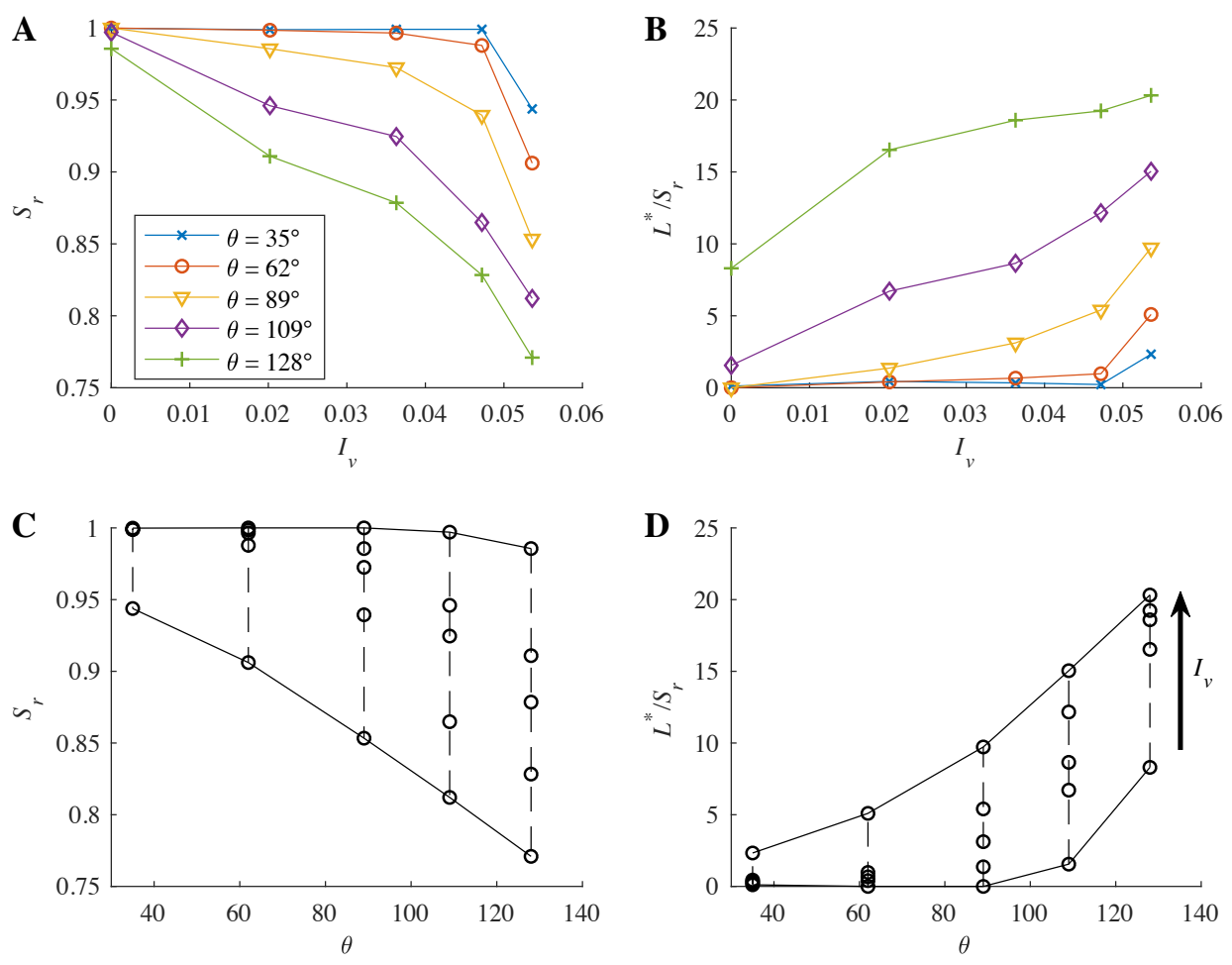

FIG. 5. (A) Final saturation of invading fluid $S_{r}$ (or displacement efficiency, which is the fraction of defending fluid that has been displaced from the media at the end of the simulation) as a function of $I_{v}$ and $\theta$. (B) Final normalized interfacial length $L^{*}$ over final saturation $S_{r}$ as a function of $I_{v}$ and $\theta$. (C) and (D) Same data set as a function of contact angle $\theta$ for different topological disorders. Black arrow indicates the direction of increasing values of $I_{v}$ from 0 to 0.054 .

(Fig. 6B). Thus, for given contact angle $\theta$, the critical angle $\alpha_{\text {crit }}$ can be calculated as $\alpha_{\text {crit }}=\min \left(\alpha_{\text {touch }}, \alpha_{\text {overlap }}\right)$, where $\alpha_{\text {touch }}$ is calculated based on $\theta$ and $\alpha_{\text {overlap }}=$ $90^{\circ}$. Different characteristic front shape indicated by $\alpha_{\text {overlap }}$ may be used by other researchers depending on the porosity of the medium [21]. In our study it is found $\alpha_{\text {overlap }}$ will not significantly impact the results and $90^{\circ}$ is adopted. Finally, for every throat, the $P_{c, \max }$ can be calculated as:

$$
P_{c, \max }=\max \left(P_{c}\right), \alpha \in\left[-90^{\circ}, \alpha_{\text {crit }}\right],
$$

which is the maximum capillary pressure before the front reaches any of the instability state at burst, touch, or overlap. It is found that log-normal curves can well fit most of the $P_{c, \text { max }}$ distributions, and the probability distributions of $P_{c, \max }$ for different $I_{v}$ and $\theta$ are plotted in Fig. 7. It shows that as the medium becomes more disordered, the distribution of $P_{c, \max }$ spreads out. In the meantime, increase in contact angle of invading fluid amplifies this effect, which further increases the variation in $P_{c, \max }$. Thus, the $P_{c, \max }$ distribution captures the interplay among wettability and geometry of the system, reflecting the resultant capillary resistance, which should have close relationship with the displacement patterns. To validate this hypothesis, the final saturation of invading fluid $S_{r}$, or displacement efficiency, is plotted against the standard deviation of $\log \left(P_{c, \max }\right)$ in Fig. 8 for all our simulation cases. Collapses of curves for different wettability conditions and disorder can be observed, showing a linear relationship with a correlation coefficient $R=$ -0.9261 .

On the other hand, regarding the fluid-fluid interfacial length, Liu et al. [55] found that the interfacial length $L$ and saturation of invading fluid $S$ can be correlated with a power-law relationship: $L=k S^{\beta}$, where $k$ and $\beta$ are fitting parameters depending on the geometry. Here, we propose a modified correlation between interfacial length and saturation assuming a power law relation between normalized interfacial length $L^{*}$ and "injection length" $v t$ :

$$
L^{*}=\frac{L}{H}=k(v t)^{\beta}=k(\phi \cdot W \cdot S)^{\beta},
$$

where, $v$ is the injection velocity, $t$ is time, $H$ is the injection width (across which the invading fluid is injected), $\phi$ is porosity, $W$ is the domain longitudinal length, $S$ is saturation of invading fluid, $k$ and $\beta$ are parameters that can be estimated based on capillary index $I_{c}$ depending on system disorder and wettability conditions, which is defined as:

$$
I_{c}=\frac{I_{v, \text { max }}-I_{v}}{I_{v, \text { max }}-I_{v, \text { min }}} \cdot \frac{\cos \left(\theta_{\max }\right)-\cos (\theta)}{\cos \left(\theta_{\max }\right)-\cos \left(\theta_{\min }\right)},
$$

where $I_{v, \max }$ is the maximum disorder index depending on overall porosity of the geometry and total number of 
A

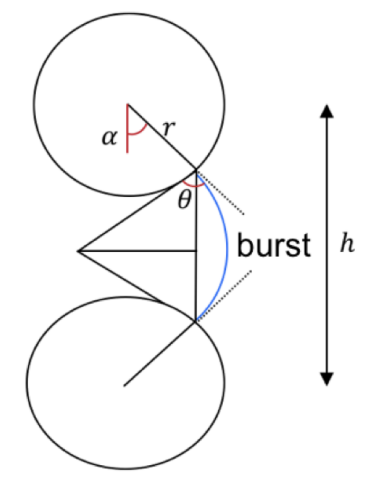

B

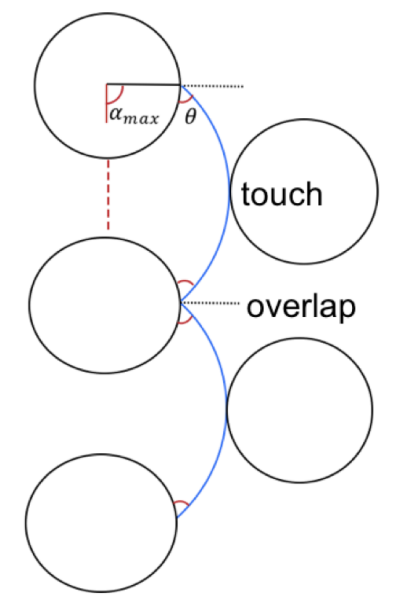

FIG. 6. (A) Schematic figure for calculation of local capillary pressure as a function of center-to-center distance of particle $h$, radius $r$, contact angle $\theta$, and filling angle $\alpha$ (B) Schematic figure for calculation of $\alpha_{\text {crit }}$, which is the maximum allowable $\alpha$, larger than which the local throat being considered (red dashed line) will be invaded.
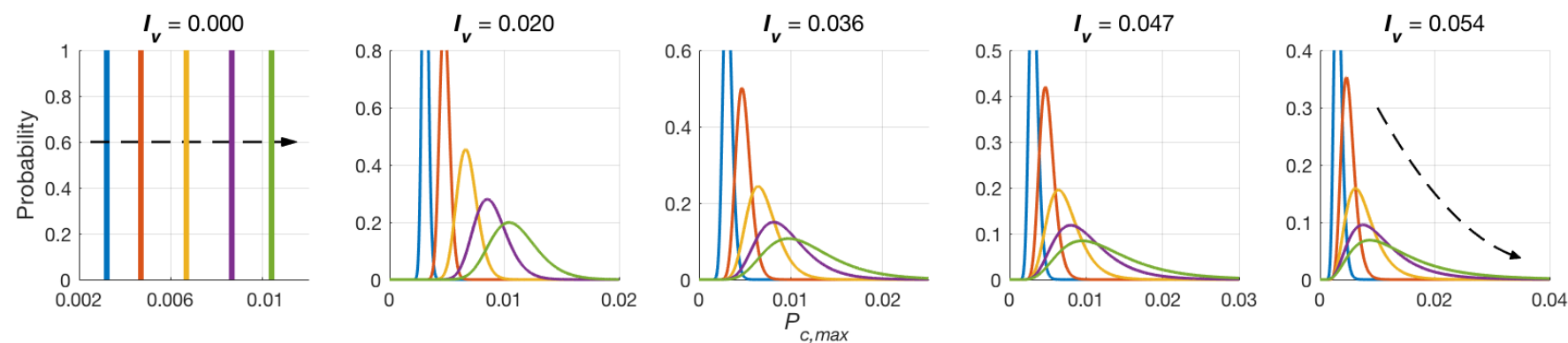

FIG. 7. Probability distribution of local maximum capillary pressure for different disorder and wettability (histogram for $I_{v}=0$ and probability density function for log-normal fitting of other values of $I_{v}$ ). Black dashed arrow indicates the direction of increasing contact angle. Blue, orange, yellow, purple, and green colors correspond to $\theta=35^{\circ}, 62^{\circ}, 89^{\circ}, 109^{\circ}, 128^{\circ}$, respectively.

obstacles inside the system, being 0.08 for the current setting. $I_{v, \min }=0$ is the disorder index for fully ordered system. $\cos \left(\theta_{\max }\right)$ and $\cos \left(\theta_{\min }\right)$ are -1 and 1 , corresponding to $\theta=180^{\circ}$ and $\theta=0^{\circ}$ respectively. As we have discussed previously that increase in contact angle and topological disorder promotes fingering, therefore, $I_{c}$ is a direct indicator of the collaborative effect due to medium geometry and wettability conditions. A small value of $I_{c}$ implies relatively large $\theta$ and $I_{v}$, which leads to less efficient displacement, while larger values of $I_{c}$ would correspond to more stable displacement. We plot the parameters $\beta$ and $\ln (k)$ from $\operatorname{Eqn}(10)$ as function of $I_{c}$ in Fig. 9A and found that with $I_{c}<0.5$, both $\beta$ and $\ln (k)$ show a strong linear relation with $I_{c}$, having $R$ of -0.9467 and 0.9958 respectively. For $I_{c} \geq 0.5$, the displacement patterns are all stable, resulting in small $\beta$ and large $\ln (k)$, indicating weak dependence of $L^{*}$ on $S_{r}: L^{*}$ is only composed of the fluid front, being almost constant during the stable displacement. In Fig. 9A, the black dashed line and a value of 0 correspond to the theoretical values for $\ln (k)$ and $\beta$ for perfect stable displacement. Clearly, it can be seen that $I_{c}$ can also be used to classify displacement patterns (9B). As shown in Fig. 3, 25 patterns are separated into 3 regions by the dashed line $\left(I_{c}=0.5\right)$ and solid line $\left(I_{c}=0.3\right)$ depending on the values of $I_{c}$. A smaller value of $I_{c}$ implies larger interfacial length per unit of saturation. Note for the left-bottom displacement pattern in Fig. 3, if zoom in and observe carefully, trapping event actually occurs at every obstacle during which small bubbles are formed, leading to much larger interfacial length between fluids than it appears to be. Overall, the proposed power law relation (Eqn (9)) together with capillary index $I_{c}$ (Eqn.(10)) provide a rigorous method to capture the quantitative relation between $L^{*}$ and $S_{r}$ for different disorder and wettability conditions, offering a reasonable way to predict the value of interfacial length.

In the current study, in order to focus on the effects of disorder and its coupling with wettability on fluid displacement in porous media, the injecting velocity in all simulations are the same, implying a constant capillary number $C a$. Although the influence of $C a$ is not investigated, based on numerous past works, since it is the uneven distribution of capillary pressure that leads to unstable displacement, a decrease in $C a$ would make all the displacement patterns shown in Fig. 3 more unstable since the capillary effect would become more significant. 


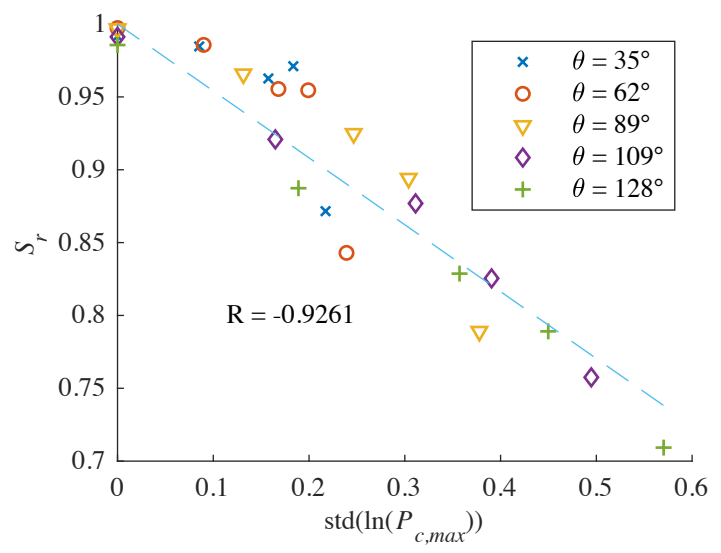

FIG. 8. Final saturation $S_{r}$ as a function of standard deviation of logarithm maximum capillary pressure for different topological disorder and wettability.
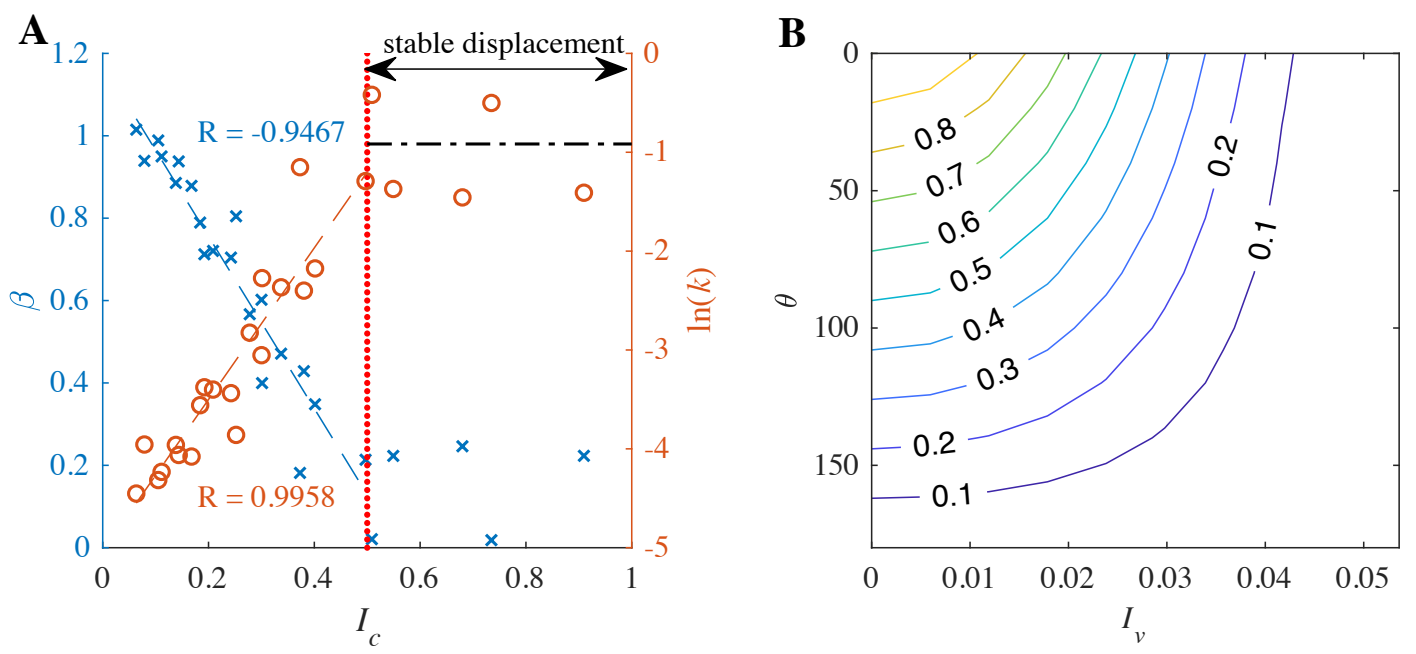

FIG. 9. (A) $\beta$ (blue cross) and $\ln (k)$ (orange circle) in Eqn. (9) as a function of $I_{c}$. The blue dashed line and orange solid line are the best fit lines for $\beta$ and $\ln (k)$ respectively. Black dashed line indicates the theoretical value for $\ln (k)$ in perfect stable displacement. (B) Contour plot of $I_{c}$ as functions of topological disorder $\left(I_{v}\right)$ and wettability $(\theta)$.

We also limit our attention to situations where SaffmanTaylor instability, or viscous fingering, is not present by setting the viscosity ratio $M=0.8$.

\section{CONCLUSION}

We systematically study the impact of topological disorder and its coupling with wettability on multiphase flow in porous media via fluid-fluid displacement simulation using Lattice Boltzmann method. It has been shown that the disorder of porous media and wettability play a significant role on the fluid-fluid displacement patterns. In addition to the overall porosity of the medium, the consideration of an appropriate "disorder index" is required to capture the effects of micro-structure on fluid displacement. The modified disorder index $I_{v}$ is able to characterize geometries with different disorder by reflect- ing the degree of fluctuation of local porosity based on Voronoi diagram. Our results show larger contact angle and increasing disorder promote fingering, leading to larger fluid-fluid interfacial area and lower displacement efficiency. To analytically investigate how wettability and disorder collaboratively influence displacement, we calculate the maximum allowable local capillary pressure $P_{c, \max }$ based on three pore-scale mechanisms during displacement: burst, touch, and overlap. It is found that the standard deviation of $\log \left(P_{c, \max }\right)$ strongly correlates with the displacement efficiency for all wettability conditions and disorder. We proposed a more general power law relation and defined the capillary index $I_{c}$, which offers a rigorous way to capture the quantitative relation between $L^{*}$ and $S_{r}$ for different disorder and wettability conditions.

While in current work we only consider obstacles of same size, for media with different sizes of grains, mod- 
ified Voronoi diagram can be adopted to generate the disorder index by taking the varying radii into account. We provide qualitative and quantitative insight into how geometrical features and wettability conditions collaboratively impact the fluid displacement, paving the way for further study of disorder and wettability control on multiphase flow in porous media.

\section{ACKNOWLEDGEMENT}

This work was financially supported by Australian Research Council (Projects DP170102886) and The University of Sydney SOAR Fellowship. YG aknowledges the financial support of Labex MMCD(ANR-11-LABX-02201) for his stay at Laboratoire Navier at ENPC. This research was undertaken with the assistance of the HPC service at The University of Sydney.
[1] Michael L. Szulczewski, Christopher W. MacMinn, Howard J. Herzog, and Ruben Juanes, "Lifetime of carbon capture and storage as a climate-change mitigation technology," Proceedings of the National Academy of Sciences 109, 5185-5189 (2012).

[2] Juerg M. Matter, Martin Stute, Sandra Ó. Snæbjörnsdottir, Eric H. Oelkers, Sigurdur R. Gislason, Edda S. Aradottir, Bergur Sigfusson, Ingvi Gunnarsson, Holmfridur Sigurdardottir, Einar Gunnlaugsson, Gudni Axelsson, Helgi A. Alfredsson, Domenik Wolff-Boenisch, Kiflom Mesfin, Diana Fernandez de la Reguera Taya, Jennifer Hall, Knud Dideriksen, and Wallace S. Broecker, "Rapid carbon mineralization for permanent disposal of anthropogenic carbon dioxide emissions," Science 352, 1312-1314 (2016).

[3] L.W. Lake and Society of Petroleum Engineers (U.S.), Fundamentals of Enhanced Oil Recovery, SPE continuing education (SPE, 1986).

[4] Martin Blunt, F.John Fayers, and Franklin M. Orr, "Carbon dioxide in enhanced oil recovery," Energy Conversion and Management 34, 1197 - 1204 (1993), proceedings of the International Energy Agency Carbon Dioxide Disposal Symposium.

[5] Farhad Nadim, George E Hoag, Shili Liu, Robert J Carley, and Peter Zack, "Detection and remediation of soil and aquifer systems contaminated with petroleum products: an overview," Journal of Petroleum Science and Engineering 26, 169 - 178 (2000).

[6] J. Lipiec, J. KuA, A. SowiAska-Jurkiewicz, and A. Nosalewicz, "Soil porosity and water infiltration as influenced by tillage methods," Soil and Tillage Research 89, 210 220 (2006).

[7] Bo Dong, Y. Y. Yan, Weizhong Li, and Yongchen Song, "Lattice boltzmann simulation of viscous fingering phenomenon of immiscible fluids displacement in a channel," Computers \& Fluids 39, 768-779 (2010).

[8] Changyong Zhang, Mart Oostrom, Thomas W. Wietsma, Jay W. Grate, and Marvin Warner, "Influence of viscous and capillary forces on immiscible fluid displacement: Pore-scale experimental study in a water-wet micromodel demonstrating viscous and capillary fingering," Energy \& Fuels - ENERG FUEL 25, 3493-3505 (2011).

[9] Haihu Liu, Albert J. Valocchi, Qinjun Kang, and Charles Werth, "Pore-scale simulations of gas displacing liquid in a homogeneous pore network using the lattice boltzmann method," Transport in Porous Media 99, 555-580 (2013).

[10] Marek Cieplak and Mark O. Robbins, "Influence of contact angle on quasistatic fluid invasion of porous media," Phys. Rev. B 41, 11508-11521 (1990).
[11] B. Dong, Y. Y. Yan, W. Z. Li, and Y. C. Song, "Simulation of the influence of surface wettability on viscous fingering phenomenon in porous media," Journal of Bionic Engineering 7, 267-275 (2010).

[12] Benzhong Zhao, Christopher W. MacMinn, and Ruben Juanes, "Wettability control on multiphase flow in patterned microfluidics," Proceedings of the National Academy of Sciences 113, 10251-10256 (2016).

[13] Jing-Den Chen and David Wilkinson, "Pore-scale viscous fingering in porous media," Phys. Rev. Lett. 55, 18921895 (1985).

[14] G M Homsy, "Viscous fingering in porous media," Annual Review of Fluid Mechanics 19, 271-311 (1987).

[15] Roland Lenormand, Eric Touboul, and Cesar Zarcone, "Numerical models and experiments on immiscible displacements in porous media," Journal of Fluid Mechanics 189 (1988), 10.1017/s0022112088000953.

[16] Christophe Cottin, Hugues Bodiguel, and Annie Colin, "Drainage in two-dimensional porous media: From capillary fingering to viscous flow," Phys. Rev. E 82, 046315 (2010).

[17] Celeste Odier, Bertrand Levache, Enrich SantanachCarreras, and Denis Bartolo, "Forced imbibition in porous media: A fourfold scenario," Physical Review Letters 119 (2017).

[18] Y. C. Yortsos, B. Xu, and D. Salin, "Phase diagram of fully developed drainage in porous media," Phys. Rev. Lett. 79, 4581-4584 (1997).

[19] Michael Jung, Martin Brinkmann, Ralf Seemann, Thomas Hiller, Marta Sanchez de La Lama, and Stephan Herminghaus, "Wettability controls slow immiscible displacement through local interfacial instabilities," Phys. Rev. Fluids 1, 074202 (2016).

[20] Mathias Trojer, Michael L. Szulczewski, and Ruben Juanes, "Stabilizing fluid-fluid displacements in porous media through wettability alteration," Physical Review Applied 3 (2015), 10.1103/PhysRevApplied.3.054008.

[21] Ran Holtzman and Enrico Segre, "Wettability stabilizes fluid invasion into porous media via nonlocal, cooperative pore filling," Phys. Rev. Lett. 115, 164501 (2015).

[22] Haihu Liu, Yonghao Zhang, and Albert J. Valocchi, "Lattice boltzmann simulation of immiscible fluid displacement in porous media: Homogeneous versus heterogeneous pore network," Physics of Fluids 27 (2015), $10.1063 / 1.4921611$.

[23] R. Holtzman, "Effects of pore-scale disorder on fluid displacement in partially-wettable porous media," Sci Rep 6, 36221 (2016). 
[24] Tannaz Pak, Ian B. Butler, Sebastian Geiger, Marinus I. J. van Dijke, and Ken S. Sorbie, "Droplet fragmentation: $3 \mathrm{~d}$ imaging of a previously unidentified pore-scale process during multiphase flow in porous media," Proceedings of the National Academy of Sciences 112, 19471952 (2015).

[25] Ran Holtzman and Ruben Juanes, "Crossover from fingering to fracturing in deformable disordered media," Phys. Rev. E 82, 046305 (2010).

[26] Changyong Zhang, Mart Oostrom, Jay W Grate, Thomas W Wietsma, and Marvin Warner, "Liquid co2 displacement of water in a dual-permeability pore network micromodel," Environmental science \& technology 45, 7581-8 (2011).

[27] Wei Xu, Jeong Tae Ok, Feng Xiao, Keith B. Neeves, and Xiaolong Yin, "Effect of pore geometry and interfacial tension on water-oil displacement efficiency in oil-wet microfluidic porous media analogs," Physics of Fluids 26, 093102 (2014).

[28] Jing-Den Chen and David Wilkinson, "Pore-scale viscous fingering in porous media," Phys. Rev. Lett. 55, 18921895 (1985).

[29] Martin Blunt and Peter King, "Relative permeabilities from two- and three-dimensional pore-scale network modelling," Transport in Porous Media 6, 407-433 (1991).

[30] Mohammed S. Al-Gharbi and Martin J. Blunt, "Dynamic network modeling of two-phase drainage in porous media," Phys. Rev. E 71, 016308 (2005).

[31] M. Ferer, Chuang Ji, Grant S. Bromhal, Joshua Cook, Goodarz Ahmadi, and Duane H. Smith, "Crossover from capillary fingering to viscous fingering for immiscible unstable flow: Experiment and modeling," Phys. Rev. E 70, 016303 (2004).

[32] Stanley Osher and James A Sethian, "Fronts propagating with curvature-dependent speed: Algorithms based on hamilton-jacobi formulations," Journal of Computational Physics 79, 12 - 49 (1988).

[33] Hai Huang, Paul Meakin, and Moubin Liu, "Computer simulation of two-phase immiscible fluid motion in unsaturated complex fractures using a volume of fluid method," Water Resources Research - WATER RESOUR RES 411 (2005).

[34] Ali Q. Raeini, Martin J. Blunt, and Branko Bijeljic, "Modelling two-phase flow in porous media at the pore scale using the volume-of-fluid method," Journal of Computational Physics 231, 5653 - 5668 (2012).

[35] H.A. Akhlaghi Amiri and A.A. Hamouda, "Evaluation of level set and phase field methods in modeling two phase flow with viscosity contrast through dual-permeability porous medium," International Journal of Multiphase Flow 52, 22 - 34 (2013).

[36] Harris Sajjad Rabbani, Dani Or, Ying Liu, ChingYao Lai, Nancy B. Lu, Sujit S. Datta, Howard A. Stone, and Nima Shokri, "Suppressing viscous fingering in structured porous media," Proceedings of the National Academy of Sciences (2018), 10.1073/pnas.1800729115.

[37] Wei Shyy, Richard W. Smith, H. S. Udaykumar, and Madhukar M. Rao, Computational Fluid Dynamics with Moving Boundaries (Taylor \& Francis, Inc., Bristol, PA, USA, 1996).

[38] Xiaowen Shan and Hudong Chen, "Lattice boltzmann model for simulating flows with multiple phases and components," Physical Review E 47, 1815-1819 (1993).
[39] Xiaowen Shan and Gary Doolen, "Multicomponent lattice-boltzmann model with interparticle interaction," Journal of Statistical Physics (1995).

[40] Zhaoli Guo and T. S. Zhao, "Lattice boltzmann model for incompressible flows through porous media," Phys. Rev. E 66, 036304 (2002).

[41] H.A. Akhlaghi Amiri and A.A. Hamouda, "Pore-scale modeling of non-isothermal two phase flow in $2 \mathrm{~d}$ porous media: Influences of viscosity, capillarity, wettability and heterogeneity," International Journal of Multiphase Flow 61, $14-27$ (2014).

[42] Timm Kruger, Halim Kusumaatmaja, Alexandr Kuzmin, Orest Shardt, Goncalo Silva, and Erlend Magnus Viggen, LBM - the principles and methods (Springer, 2017).

[43] F. Diotallevi, L. Biferale, S. Chibbaro, A. Lamura, G. Pontrelli, M. Sbragaglia, S. Succi, and F. Toschi, "Capillary filling using lattice boltzmann equations: The case of multi-phase flows," The European Physical Journal Special Topics 166, 111-116 (2009).

[44] Li Chen, Qinjun Kang, Yutong Mu, Ya-Ling He, and Wen-Quan Tao, "A critical review of the pseudopotential multiphase lattice boltzmann model: Methods and applications," International Journal of Heat and Mass Transfer 76, 210-236 (2014).

[45] Haihu Liu, Qinjun Kang, Christopher R. Leonardi, Sebastian Schmieschek, Ariel Narvaez, Bruce D. Jones, John R. Williams, Albert J. Valocchi, and Jens Harting, "Multiphase lattice boltzmann simulations for porous media applications," Computational Geosciences 20, 777-805 (2015).

[46] Hadrien Laubie, Farhang Radjai, Roland Pellenq, and Franz-Josef Ulm, "Stress transmission and failure in disordered porous media," Phys. Rev. Lett. 119, 075501 (2017).

[47] Oshri Borgman, Paolo Fantinel, Wieland LÃijhder, Lucas Goehring, and Ran Holtzman, "Impact of spatially correlated pore-scale heterogeneity on drying porous media," Water Resources Research 53, 5645-5658.

[48] Haibo Huang, Daniel T. Thorne, Marcel G. Schaap, and Michael C. Sukop, "Proposed approximation for contact angles in shan-and-chen-type multicomponent multiphase lattice boltzmann models," Phys. Rev. E 76, 066701 (2007).

[49] Jonas Latt and Bastien Chopard, "Lattice boltzmann method with regularized non-equilibrium distribution functions," Physics of Fluids (2005).

[50] A.A. Mohamad, LBM - fundamentals and engineering applications with computer codes (Springer, 2011).

[51] P. Raiskinmäki, A. Shakib-Manesh, A. Jäsberg, A. Koponen, J. Merikoski, and J. Timonen, "Lattice-boltzmann simulation of capillary rise dynamics," Journal of Statistical Physics 107, 143-158 (2002).

[52] Hu Ran, Wan Jiamin, Yang Zhibing, Chen Yi-Feng, and Tokunaga Tetsu, "Wettability and flow rate impacts on immiscible displacement: A theoretical model," Geophysical Research Letters 45, 3077-3086.

[53] Mathias Trojer, Michael L. Szulczewski, and Ruben Juanes, "Stabilizing fluid-fluid displacements in porous media through wettability alteration," Phys. Rev. Applied 3, 054008 (2015).

[54] Marek Cieplak and Mark O. Robbins, "Dynamical transition in quasistatic fluid invasion in porous media," Phys. Rev. Lett. 60, 2042-2045 (1988). 
[55] Haihu Liu, Albert J. Valocchi, Charles Werth, Qinjun Kang, and Mart Oostrom, "Pore-scale simulation of liquid co 2 displacement of water using a two-phase lat- tice boltzmann model," Advances in Water Resources 73 , 144-158 (2014). 